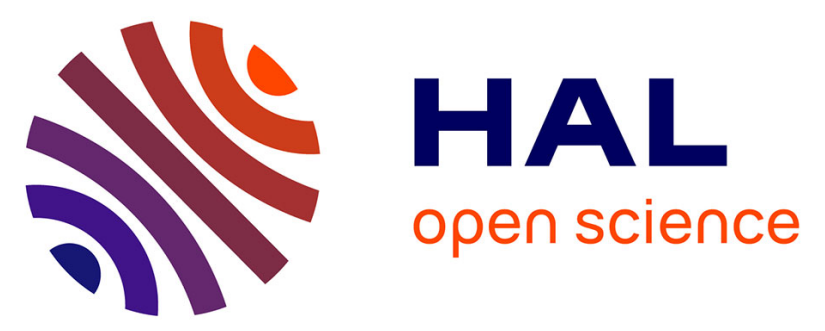

\title{
Synthesis of superheavy elements: Uncertainty analysis to improve the predictive power of reaction models
}

\author{
H. Lu, D. Boilley, Y. Abe, C. Shen
}

\section{To cite this version:}

H. Lu, D. Boilley, Y. Abe, C. Shen. Synthesis of superheavy elements: Uncertainty analysis to improve the predictive power of reaction models. Physical Review C, 2016, 94, pp.034616. 10.1103/PhysRevC.94.034616 . in2p3-01360127

\section{HAL Id: in2p3-01360127 https://hal.in2p3.fr/in2p3-01360127}

Submitted on 5 Sep 2016

HAL is a multi-disciplinary open access archive for the deposit and dissemination of scientific research documents, whether they are published or not. The documents may come from teaching and research institutions in France or abroad, or from public or private research centers.
L'archive ouverte pluridisciplinaire HAL, est destinée au dépôt et à la diffusion de documents scientifiques de niveau recherche, publiés ou non, émanant des établissements d'enseignement et de recherche français ou étrangers, des laboratoires publics ou privés. 


\title{
Synthesis of superheavy elements: Uncertainty analysis to improve the predictive power of reaction models
}

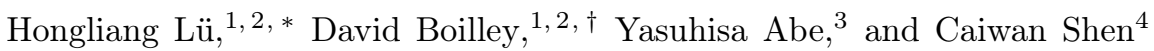 \\ ${ }^{1}$ GANIL, CEA/DRF-CNRS/IN2P3, BP 55027, F-14076 Caen cedex 5, France \\ ${ }^{2}$ Normandie Univ, UNICAEN, Caen, France \\ ${ }^{3}$ Research Center for Nuclear Physics (RCNP), Osaka University, \\ 10-1 Mihogaoka, Ibaraki, 567-0047 Osaka, Japan \\ ${ }^{4}$ School of Science, Huzhou University, Huzhou, 313000 Zhejiang, People's Republic of China
}

(Dated: September 21, 2016)

\begin{abstract}
Background: Synthesis of super-heavy elements is performed by heavy-ion fusion-evaporation reactions. However, fusion is known to be hindered with respect to what can be observed with lighter ions. Thus some delicate ambiguities remain on the fusion mechanism that eventually lead to severe discrepancies in the calculated formation probabilities coming from different fusion models.
\end{abstract}

Purpose: In the present work, we propose a general framework based upon uncertainty analysis in the hope of constraining fusion models.

Method: To quantify uncertainty associated with the formation probability, we propose to propagate uncertainties in data and parameters using the Monte-Carlo method in combination with a cascade code called KEWPIE2, with the aim of determining the associated uncertainty, namely the $95 \%$ confidence interval. We also investigate the impact of different models or options, which cannot be modeled by continuous probability distributions, on the final results. An illustrative example is presented in detail and then a systematic study is carried out for a selected set of cold-fusion reactions.

Results: It is rigorously shown that, at the $95 \%$ confidence level, the total uncertainty of the empirical formation probability appears comparable to the discrepancy between calculated values.

Conclusions: The results obtained from the present study provide a direct evidence for predictive limitations of the existing fusion-evaporation models. It is thus necessary to find other ways to assess such models for the purpose of establishing a more reliable reaction theory, which is expected to guide future experiments on the production of super-heavy elements.

PACS numbers: 25.70.Jj, 24.10.-i, 24.60.Dr, 06.20.-f

\section{INTRODUCTION}

Experimentally the synthesis of super-heavy elements (SHE) is realized by means of the so-called fusionevaporation reaction which, from a theoretical viewpoint based upon the Bohr independence hypothesis [1], is divided into two separate phases:

- The fusion phase, during which a nucleus-nucleus collision may lead to the formation of an excited heavy nucleus.

- The de-excitation phase, where the newlyformed heavy nucleus has to be chilled by evaporation of light particles, including $\gamma$-ray emission against nuclear fission.

For the latter, it is often described using a cascade code. In our case, we shall employ the KEWPIE2 code [2, 3]. Regarding the fusion process, it is decomposed into two successive steps [4]:

\footnotetext{
* Current address: Neutron Metrology and Dosimetry Laboratory, IRSN, BP 3, 13115 Saint-Paul-lez-Durance Cedex, France

$\dagger$ boilley@ganil.fr
}

- The capture step, corresponding to passing over the Coulomb (or Bass [5]) barrier, after which two colliding nuclei stick together.

- The formation step, from the contact configuration to the compound nucleus $(\mathrm{CN})$. During this step, the system has to overcome an inner potential barrier, which results in the creation of a compound system, where the incident particle combines with the target nucleus and the total energy is shared among all the confined nucleons. Here, it should be mentioned that the formation step was introduced to explain the so-called fusion hindrance phenomenon that only occurs in heavy reaction systems (with a charge product of the projectile-target combination $\left.Z_{p} Z_{t} \gtrsim 1600-1800\right)$. Regarding lighter systems, the inner barrier should no longer exist and the colliding nuclei automatically fuse after the capture step.

It should be noted that the main contribution to the fusion hindrance in heavy-ion collisions is due to the formation step, consisting of a diffusion process over an inner potential barrier. Over the past two decades, qualitative features of the formation dynamics have been well understood within the framework of the Langevin equation 6 12]. This formalism is not unique and some se- 
rious ambiguities remain on the inner-barrier height and the dissipation strength. Moreover, during an experiment, fusion-fission events that have reached the compound state and quasi-fission ones that re-separate after crossing the Coulomb barrier are usually very difficult to distinguish from each other. As a consequence, this leads to a lack of reliable data on the fusion cross-sections or formation probabilities that could be employed to assess the fusion models. Another delicate issue is that, at the present time, the two separate steps of the fusion process cannot be treated within a unified framework. This remains a severe problem that could eventually affect the final results.

Precise theoretical predictions of evaporation-residue (ER) cross-sections are crucially important for conducting experiments on the synthesis of SHE, because in most cases production cross-sections are extremely low (of the order of one picobarn). For a recent experimental review, see Ref. [13. A small change of the cross-section could mean months of experiments. To this extent, the first crucial question one might ask is, how exactly can we predict evaporation-residue (ER) cross-sections with current models?

Actually, there have been a number of theoretical calculations on ER cross-sections, which appear to agree remarkably well with the measured ER cross-sections. This, of course, can be regarded as a significant theoretical achievement considering that the measured crosssections usually span at least six orders of magnitude. In the present study, we only focus on the so-called coldfusion reaction, where only one neutron can be evaporated from the compound nucleus. In this case, typical calculations on the ER cross-sections for a selected set of cold-fusion reactions are displayed in Fig. 1 taken from Ref. [14]. The experimental measurements are nicely fitted by almost all models within less than one order of magnitude. This is also the case for other models not included in this comparison such as the one based on a previous version of the code used in this study [15]. Nevertheless, if we take a closer look at the theoretical formation probabilities, as shown in Fig. 2, the calculated values can differ by two or three orders of magnitude, even though all the fusion-evaporation models seem able to reasonably fit the measured data. The large uncertainty in the calculated formation probability is not a surprise because of some serious ambiguities in the reaction mechanism of fusion dynamics, as mentioned before. However, the capture and de-excitation steps are both considered to be better known from a theoretical viewpoint and can be relatively well described on the basis of knowledge about lighter reaction systems. How come the better-known parts can accommodate large discrepancies between the calculated formation probabilities and thus reproduce the measured data? What if the experimental data are not available? To this extent, the predictive capability of the fusion model appears to be quite limited.

To establish a reliable theory for guiding future experiments on the synthesis of SHE, it is necessary to find

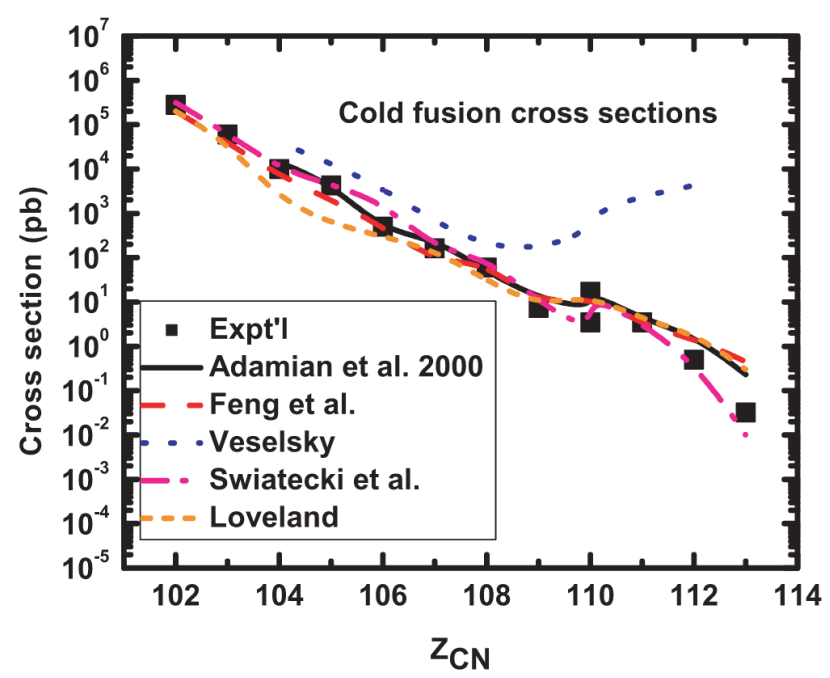

FIG. 1. [Color online] Theoretical calculations of ER crosssections for the one-neutron evaporation channel of coldfusion reactions. The calculated results can be found in Refs. [10, 16 19]. The figure is taken from Ref. [14].

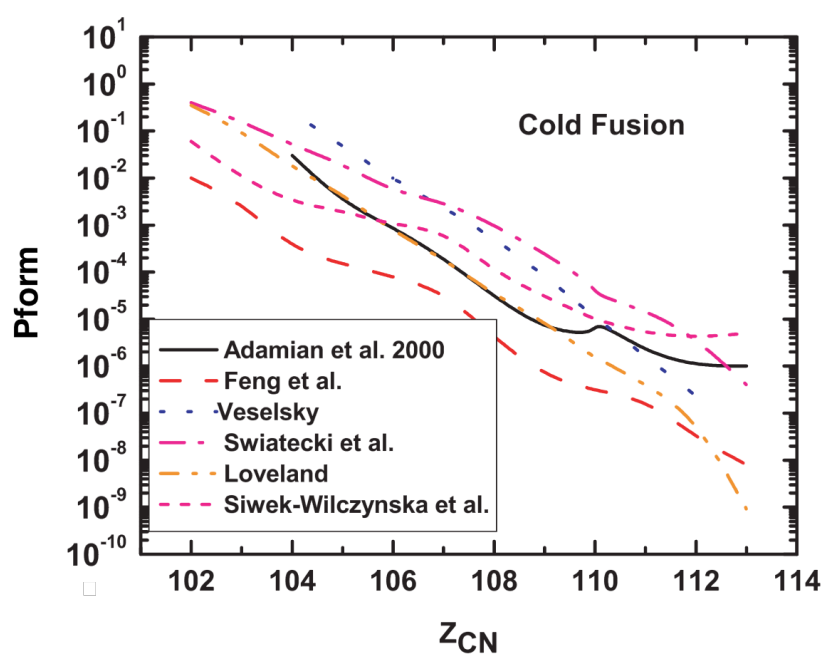

FIG. 2. [Color online] Calculated formation probabilities for the selected set of cold-fusion reactions. The theoretical results can be found in Refs. [10, 16, 20]. The figure is adapted from Ref. [14.

new methods to constrain existing fusion models with a special focus on the formation step. Thus, we perform an uncertainty analysis of what are considered as the betterknown parts of the reaction, namely the capture and deexcitation steps, to constrain the lesser-known part. In this work we will include both experimental uncertainties and uncertainties due to the remaining ambiguities in modeling. For the latter, this includes input parameters and models or approximations. The main aim of the present paper is to employ some state-of-the-art statistical methods to quantify different uncertainty sources and look more closely at their impact on the formation 
probability.

Over the past few decades, the importance of uncertainty analysis in modeling has been increasingly recognized [21, especially with the enormous development of computer simulation. It is mainly concerned with the characterization and quantification of uncertainty in numerical applications by running a great number of trials to investigate the impact of minor differences in the input on final outcomes. This kind of study is essential when one has very poor information about the input. For instance, both the free parameters and the various theoretical models used for capture and evaporation phases are usually not unambiguously determined [2, 3].

Before entering into details about uncertainty analysis, let us first briefly present the theoretical models used in this study.

\section{THEORETICAL FRAMEWORK}

It is commonly known that, based upon the Bohr independence hypothesis [1, the ER cross-section for a fusion-evaporation reaction can be explicitly expressed as

$$
\sigma_{\mathrm{ER}}\left(E_{\mathrm{cm}}\right)=\sum_{J_{C} \geq 0}^{J_{\mathrm{max}}} \sigma_{\text {fus }}\left(E_{\mathrm{cm}}, J_{C}\right) P_{\mathrm{sur}}\left(E_{C}^{*}, J_{C}\right),
$$

recalling that the relationship between incident energy in the center-of-mass frame $E_{\mathrm{cm}}$ and total excitation energy of the CN $E_{C}^{*}$ is simply given by $E_{C}^{*}=E_{\mathrm{cm}}+Q$ with $Q$ being the $Q$-value evaluated from ground-state masses. It should be noted that Eq. (1) takes into account all partial-wave contributions to the total crosssection, ending up with an upper limit, say $J_{\max }$, which can be approximately determined once the partial-wave cross-section vanishes.

\section{A. A simplified model}

In the case of SHE, the maximum spin $J_{\max }$ is essentially related to the survival probability $P_{\text {sur }}\left(E_{C}^{*}, J_{C}\right)$ which does not vanish only in the vicinity of $J_{C} \simeq$ 0 . Hence, one has $P_{\text {sur }}\left(E_{C}^{*}, J_{C}\right) \simeq P_{\text {sur }}\left(E_{C}^{*}\right)$, where $P_{\text {sur }}\left(E_{C}^{*}\right)$ can be estimated by means of the WeisskopfEwing model, and the total ER cross-section can thus be approximated as

$$
\sigma_{\mathrm{ER}}\left(E_{\mathrm{cm}}\right) \simeq \sigma_{\text {fus }}\left(E_{\mathrm{cm}}\right) P_{\mathrm{sur}}\left(E_{C}^{*}\right) .
$$

Moreover, for heavy fusing systems leading to the formation of SHE, the fusion probability needs to be rewritten as a product of the capture and formation probabilities. Accordingly, the above approximate formula can be further transformed into

$$
\sigma_{\mathrm{ER}}\left(E_{\mathrm{cm}}\right) \simeq \sigma_{\mathrm{cap}}\left(E_{\mathrm{cm}}\right) P_{\mathrm{form}}\left(E_{\mathrm{cm}}\right) P_{\mathrm{sur}}\left(E_{C}^{*}\right),
$$

where $P_{\text {form }}\left(E_{\mathrm{cm}}\right)$ stands for the formation probability due to the fusion hindrance. We should be aware that this simplified version of Eq. (1) has been widely employed to estimate ER cross-sections in many recent studies [10, 14, 19, 20, due to its simplicity.

As mentioned in the introduction, we only focus on the one-neutron evaporation channel. In this case, the ER cross-section is simply given by

$$
\sigma_{\mathrm{ER}}^{1 n} \simeq \sigma_{\text {cap }} P_{\text {form }} P_{\text {sur }}^{1 n} .
$$

It should be recalled that there is a huge discrepancy between different theoretical results on $P_{\text {form}}$. On the one hand, it is difficult to reliably measure this quantity as the identification of quasi-fission events remains a delicate issue. On the other hand, as previously mentioned, serious ambiguities in the fusion mechanism still persist. Thus, the formation probability, which is very specific to heavy systems, is considered to be the most poorly known quantity in Eq. (4).

In the following we shall attempt to constrain the formation probability by deducing it from experimental data,

$$
P_{\text {form }} \simeq \frac{\sigma_{\mathrm{ER}}^{1 n}}{\sigma_{\mathrm{cap}} P_{\mathrm{sur}}^{1 n}} .
$$

In this study, we will estimate the uncertainty of $P_{\text {form }}$ and compare it to the theoretical values calculated with various models as displayed in Fig. 2, Note that in the r.h.s. of the above equation, the numerator directly comes from experiments and the denominator from models. For the latter, we shall use the KEWPIE2 code specially dedicated to the synthesis of super-heavy elements [3.

\section{B. Capture cross section}

The capture cross-section corresponds to the Coulomb barrier crossing or to the sum of quasi-fission, fusionfission and ER cross-sections. There are several models that can be tested against some measurements. According to a review by Loveland [22] these models are able to describe the magnitudes of the capture cross sections within $50 \%$. However, the ratio of calculated to measured cross sections for various systems and models spans from 0.55 to 2.24 .

In this work we did not perform an extended analysis of the capture cross-section but picked up two typical models that are widely used and implemented as default options in the KEWPIE2 code, namely the empirical barrier-distribution (EBD) method [10, 23] and the Wentzel-Kramers-Brillouin (WKB) approximation with a proximity potential [24, 25]. In the former model, the barrier distribution is supposed to be a Gaussian with a mean value and width adjusted to reproduce a systematics of 48 reactions, whereas in the latter proximity potential parameters were adjusted to reproduce experimental data without any coupling to other channels. 


\section{Survival probability}

The survival probability of an excited heavy nucleus results from the competition between neutron evaporation and fission that are governed by the neutron-evaporation width $\Gamma_{n}$ and fission-decay width $\Gamma_{f}$, respectively.

On the one hand, the neutron-evaporation width is estimated using the Weisskopf-Ewing theory [26 28]:

$$
\Gamma_{n}\left(E_{C}^{*}\right)=\frac{\left(2 s_{n}+1\right) \mu_{n}}{\pi^{2} \hbar^{2}} \int_{0}^{E_{C}^{*}-S_{n}} \frac{\sigma_{\mathrm{inv}}^{n}\left(\epsilon_{n}\right) \rho_{B}\left(E_{B}^{*}\right)}{\rho_{C}\left(E_{C}^{*}\right)} \epsilon_{n} \mathrm{~d} \epsilon_{n},
$$

where $C$ denotes a compound nucleus at excitation energy $E_{C}^{*}$ and $B$ the daughter nucleus at excitation energy $E_{B}^{*}=E_{C}^{*}-S_{n}-\epsilon_{n}$, where $S_{n}$ is the binding energy of neutrons in the compound nucleus and $\epsilon_{n}$ the kinetic energy of evaporated neutrons in the center-of-mass frame. $\mu_{n}$ is the reduced mass of the reaction system. For neutrons, the cross section for the time-reversed reaction $\sigma_{\text {inv }}^{n}$ is given by

$$
\sigma_{\text {inv }}^{n}\left(\epsilon_{n}\right)=g_{0}\left(1+\frac{g_{1}}{\epsilon_{n}}\right) \pi \tilde{R}^{2}
$$

where $g_{0}=0.76+1.93 A_{B}^{-1 / 3}, g_{0} g_{1}=1.66 A_{B}^{-2 / 3}-0.05$ and $\tilde{R}=1.7 A_{B}^{1 / 3}$ fm 29 .

On the other hand, the fission-decay width is usually calculated within the Bohr-Wheeler (BW) transitionstate method 30]:

$$
\Gamma_{f}^{\mathrm{BW}}\left(E_{C}^{*}\right)=\frac{1}{2 \pi \rho_{C}^{\mathrm{gs}}\left(E_{C}^{*}\right)} \int_{0}^{E_{C}^{*}-B_{f}} \rho_{C}^{\mathrm{sd}}\left(E_{\mathrm{sd}}^{*}\right) \mathrm{d} \epsilon_{f},
$$

where the excitation energy at the saddle point $E_{\mathrm{sd}}^{*}$ is equal to $E_{\mathrm{C}}^{*}-B_{f}-\epsilon_{f}$ with $\epsilon_{f}$ being the kinetic energy of the collective motion. Here, $B_{f}$ represents the fission barrier that is known to have a great influence on the stability of super-heavy elements. A recent review 31. shows that this quantity is difficult to estimate and large discrepancies that can reach up to several $\mathrm{MeV}$ still remain in the predicted values.

In this work, we first use a common approximation: $B_{f} \simeq B_{\mathrm{LDM}}-\Delta E_{\mathrm{sh}}$ with $B_{\mathrm{LDM}}$ and $\Delta E_{\text {sh }}$ being respectively the liquid-drop fission barrier and the shellcorrection energy in the ground state. We also considered a table based upon a so-called microscopic-macroscopic theory [32]. Note that the shell-correction energy is the same in both cases.

From a dynamical point of view, the fission-decay width evaluated by Eq. (8) can actually be refined by introducing the Kramers factor 33],

$$
K=\sqrt{1+\left(\frac{\beta}{2 \omega_{\mathrm{sd}}}\right)^{2}}-\frac{\beta}{2 \omega_{\mathrm{sd}}}
$$

and the Strutinsky factor [34,

$$
S=\frac{\hbar \omega_{\mathrm{gs}}}{T_{\mathrm{gs}}}
$$

In the above factors, $\beta$ stands for the reduced friction coefficient that takes into account the effect of viscosity on the fission process. Its value is not well known. $\hbar \omega_{\mathrm{gs}}$ and $\hbar \omega_{\text {sd }}$ denote the potential curvature in the ground state and at the saddle point respectively. They are both fixed at $1.0 \mathrm{MeV}$. Finally, the total fission-decay width is given by

$$
\Gamma_{f}=K \cdot S \cdot \Gamma_{f}^{\mathrm{BW}} .
$$

In the following study, the product $K \cdot S$ is referred to as the Kramers-Strutinsky factor. Some models take it into account; some others do not.

The state density $\rho$ enters both decay widths in Eqs. (6) and (8). In our model, the intrinsic state-density formula for a nucleus comprising two kinds of particles, namely protons and neutrons, is explicitly given by 35

$$
\begin{aligned}
\rho_{\text {int }}\left(E^{*}\right) & =\frac{\sqrt{\pi}}{12} \frac{\exp \left(\beta_{0} E^{*}+a / \beta_{0}\right)}{\sqrt{\beta_{0} E^{* 3}}}\left(\frac{g_{0}^{2}}{4 g_{n} g_{p}}\right)^{1 / 2} \\
& \times \frac{1-\exp \left(-a / \beta_{0}\right)}{\left[1-\frac{1}{2} E^{*} \beta_{0} \exp \left(-a / \beta_{0}\right)\right]^{1 / 2}},
\end{aligned}
$$

where $g_{n}$ and $g_{p}$ are respectively the neutron and proton single-particle state densities at the Fermi energy. Here, $g_{0}=g_{n}+g_{p}$ and typically, one has the following approximation: $g_{n} \simeq g_{p}$. In this study, we shall consider various formulas for the level density parameter. In addition to the Tōke-Świątecki model [36] chosen as default value, we also selected Reisdorf and Pomorska et al.'s models [24, 37.

Ignatyuk's prescription was also taken into consideration for the energy dependence of the level-density parameter $a$ in the ground state [38]:

$$
a_{\mathrm{gs}}\left(E^{*}\right)=a_{\mathrm{gs}}\left[1+\left(1-e^{-E^{*} / E_{d}}\right) \frac{\Delta E_{\mathrm{sh}}}{E^{*}}\right],
$$

where $E_{d}$ represents the shell-damping factor that characterizes how fast the shell effect is disappearing with excitation energy. Its value is not well established.

Moreover, the intrinsic state density should be enhanced by a certain factor as a function of excitation energy $E^{*}$, namely

$$
\rho\left(E^{*}\right)=\rho_{\text {int }}\left(E^{*}\right) \kappa_{\text {coll }}\left(E^{*}\right) .
$$

For more details on the collective enhancement factor $\kappa_{\text {coll }}$ used in KEWPIE2, the reader is referred to Ref. [3]. This factor is not always implemented in models used to study the survival probability of super-heavy elements.

\section{Average values due to the loss of beam energy}

Here, we only concentrate on the survival probability with respect to the $1 n$-channel $P_{\text {sur }}^{1 n}$, which can be written in the following form:

$$
P_{\mathrm{sur}}^{1 n}=P_{\mathrm{sur}}^{1 n}\left(E^{*}, \beta, E_{d}\right) .
$$




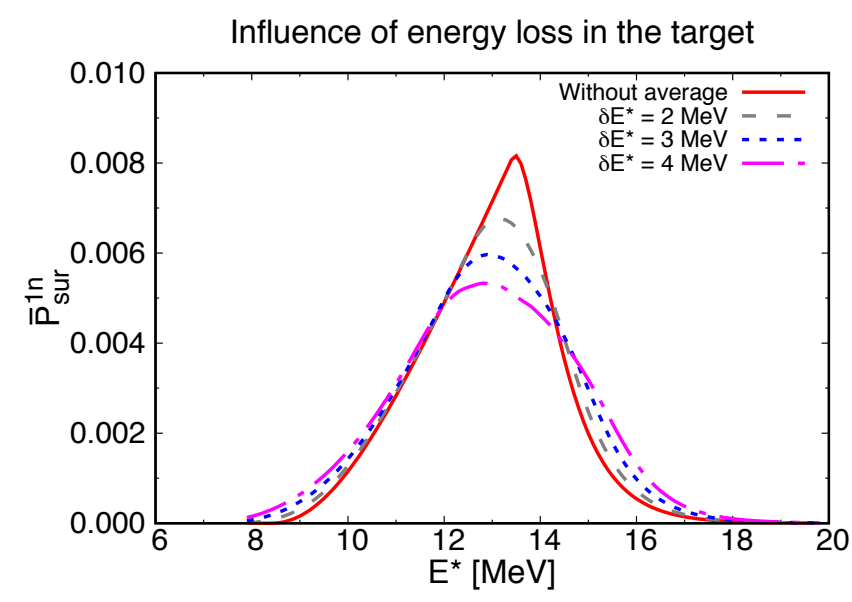

FIG. 3. [Color online] Average of the survival probability for the $1 n$-channel over different energy intervals to take into account the loss of beam energy in the target. The solid red curve indicates the calculated survival probability without taking an average.

In practice, the measured production cross-sections are usually obtained by averaging over a certain range of excitation energy, due to the resolution of the detection setup and the loss of beam energy in the target. When the calculation is confronted with experimental data, this dispersion effect would be crucially important in the case of SHE formed via cold-fusion reactions, because the survival probability is rather peaked, as illustrated in Fig. 3 . Therefore, to be close to experimental observables, it is common to take an average over a fixed energy width $\delta E^{*}$, as proposed in Ref. [10. Thus, the average survival probability can be defined as

$$
\bar{P}_{\mathrm{sur}}^{1 n}=\frac{1}{\delta E^{*}} \int_{E_{C}^{*}-\delta E^{*} / 2}^{E_{C}^{*}+\delta E^{*} / 2} P_{\mathrm{sur}}^{1 n}\left(E^{*}, \beta, E_{d}\right) \mathrm{d} E^{*},
$$

which is also dependent on the energy loss $\delta E^{*}$ in addition to the model parameters. To carry out the integral, we employ the Gauss-Legendre quadrature method, with a number of abscissas chosen to be 12 to get a numerical precision of less than $1.0 \%$.

\section{UNCERTAINTY ANALYSIS}

In this section, we concretely formulate the problem we are dealing with and specify remaining ambiguities in the input and options of the model.

\section{A. Modeling the physical system}

The main purpose of a numerical simulation is to establish an abstract mathematical model for describing some basic features of the physical system under study.
In a general way, a physical system can be abstracted as a set of multivariate real functions, namely

$$
\begin{aligned}
\mathcal{M}: & \mathbb{R}^{N} \rightarrow \mathbb{R}^{M} \\
& \boldsymbol{x} \rightarrow \boldsymbol{y}=\mathcal{M}(\boldsymbol{x}),
\end{aligned}
$$

together with some constraints or restrictions so that the subsequent derivations would make sense. Here, the input parameters of the model can be represented by a $N$-dimensional vector $\boldsymbol{x} \in D \subset \mathbb{R}^{N}$, where $D$ denotes the domain of a model function. The vector of $M$ output quantities (or response variables) is thus a $M$ dimensional vector function. In rare cases, the model might be a simple closed-form function. But more generally, it could correspond to a black-box function, such as a computer program, which requires $N$ input values and then yields some numerical results. It should be mentioned that the model itself can also be changed so that sometimes one has different model functions for the same issue.

In our case, KEWPIE2 is employed to model the better-known parts, namely the capture cross-section and survival probability. The main input parameters for the decay part are nothing else but the reduced friction parameter $\beta$ and the shell-damping energy $E_{d}$. It should be noted that the fission barrier has been considered to be model-dependent, instead of a free parameter. In addition to the input parameters, we have also incorporated several sub-models into the code [3].

The main objective here is to extract the empirical formation probability which is simply a scalar-valued model function. Based upon Eq. (5), the model function $y=\mathcal{M}(\boldsymbol{x})$ can be constructed. Accordingly, the empirical formation probability is given as follows:

$$
\bar{P}_{\text {form }}=\frac{\sigma_{\exp }^{1 n}}{\sigma_{\text {cap }} \bar{P}_{\text {sur }}^{1 n}}=\bar{P}_{\text {form }}\left(\delta E^{*}, \beta, E_{d}, \sigma_{\exp }^{1 n}\right),
$$

where the measured data are assumed to be normally distributed. It should be noted that, sometimes, uncertainty intervals associated with the experimental data can be asymmetric. In this case, the method presented here is still valid, provided that the corresponding distribution function is available.

\section{B. Identifying and quantifying uncertainty sources}

The common uncertainty sources can be roughly summarized as follows [39]:

- Parameter uncertainty, which comes from the input parameters of the model whose values cannot be exactly inferred, neither theoretically nor experimentally. In our case, for example, the reduced friction coefficient and the shell-damping energy cannot be determined with certainty [38, 40,42. Hence, one needs to construct some input probability distributions for them. 


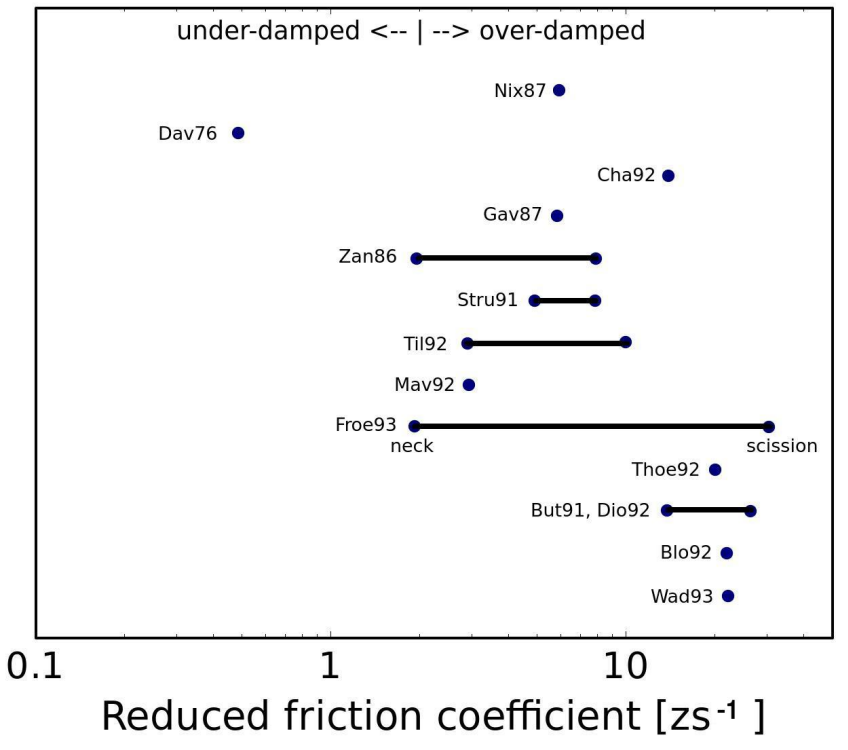

FIG. 4. Typical values of the reduced friction parameter that can be found in the literature. The figure is adapted from Ref. [1].

- Model uncertainty, which results from the lack of knowledge about the underlying true physics. Thus, it is dependent upon how accurately a model describes the true system in a realistic situation, knowing that models are only rough approximations of reality. In the present study, for example, to estimate the capture cross-section, two approximate methods are employed. Here, to assess model uncertainty, we simply investigate the effects of changing models on the final outcomes.

- Experimental uncertainty, which is directly related to the precision and accuracy of measurements. In simple cases, experimental data can be described by a Gaussian distribution.

- Numerical uncertainty, which arises because of errors corresponding to the implementation of computational methods. We should be aware that most models are too complicated to be solved analytically, so that doing a certain approximation is often necessary. This contribution should be negligible whenever possible.

It should be noted that the first two uncertainty sources are essentially related to theoretical modeling and the distinction between them is sometimes blurred, since a model can also comprise a set of parameters.

The next step consists in identifying different uncertainty sources that might come up in various contexts with the aim of quantifying them using probabilistic methods.

Regarding parameters, a sensitivity analysis introduced in our previous studies [2, 3] allowed us to extract the most relevant ones, namely the friction coefficient $\beta$ and the shell-damping energy, $E_{d}$. Their typical ranges of values can be determined by doing an exhaustive literature search. Since the choice of ranges is somewhat subjective, we would like to introduce two kinds of cases, namely the pessimistic and optimistic hypotheses. In the former one, the ranges of values are summarized as follows:

- The reduced friction coefficient $\beta \sim 1.0-9.0 \mathrm{zs}^{-1}$.

- The shell-damping energy $E_{d} \sim 13.0-25.0 \mathrm{MeV}$.

As its name indicates, such ranges are able to cover most of values that can be found in the literature [38, 40 46], but the associated uncertainties appear quite large. To justify this choice, Fig. 4 illustrates typical values of $\beta$ according to previous studies. Here, the points or bars indicate the values extracted from experimental data. It can be seen that the chosen range of $\beta$ can accommodate most of the extracted values. As regards the shell-damping energy $E_{d}$, typical values can be found in some recent papers [42, 47, which demonstrate the chosen range of values. Accordingly, the default values of the above two parameters are taken to be $5.0 \mathrm{zs}^{-1}$ and 19.0 $\mathrm{MeV}$, namely their mean values. By contrast, in the optimistic case, the above ranges are expected to be better constrained so that they could become much narrower. In view of this, some smaller ranges of values are worth taking into account, say reduced by $50 \%$, namely

- The reduced friction coefficient $\beta \sim 3.0-7.0 \mathrm{zs}^{-1}$.

- The shell-damping energy $E_{d} \sim 16.0-22.0 \mathrm{MeV}$.

This optimistic hypothesis is also inspected in the following study.

Regarding the energy loss in the target $\delta E^{*}$, it is assumed to lie in the range $2-4 \mathrm{MeV}$ [48]. Unlike the excitation energy, uncertainty in the beam energy can be safely neglected because the typical relative uncertainty is only a few percents at most.

To specify the probability density function (PDF) associated with each parameter, one needs to take into account all available information. Here, it should be noted that, in addition to the normalization condition, only the lower and upper limits are available (not that exactly). The maximum entropy principle [49, 50] would give a complicated probability distribution as detailed in Ref. [51. However, for the sake of simplicity, we stick here to the uniform probability distribution denoted as $U\left(l_{\min }, l_{\max }\right)$ in the following study.

In addition to the parameters, it should be recalled that using different models can also have an impact on the final results. This influence needs to be taken into account during analysis.

The experimental data employed in this study are mainly taken from Refs. [52 54 and are assumed to be normally distributed, namely $N\left(\mu, \sigma^{2}\right)$ with the mean value $\mu$ and the variance $\sigma^{2}$. Therefore, we are only interested in the ones with symmetric uncertainty bars, even 
TABLE I. Experimental data for the $1 n$-channel with the optimum energy and the corresponding maximal ER crosssection. The data are taken from Refs. [52,54].

\begin{tabular}{ccccc}
\hline \hline Reaction & $Z_{C}$ & $A_{C}$ & $E_{\mathrm{cm}}[\mathrm{MeV}]$ & $\max \sigma_{\exp }^{1 n}[\mathrm{pb}]$ \\
\hline${ }^{208} \mathrm{~Pb}\left({ }^{50} \mathrm{Ti}, 1 n\right){ }^{257} \mathrm{Rf}$ & 104 & 258 & 185.02 & $10419_{-1284}^{+1284}$ \\
${ }^{209} \mathrm{Bi}\left({ }^{50} \mathrm{Ti}, 1 n\right){ }^{258} \mathrm{Db}$ & 105 & 259 & 187.50 & $2200_{-240}^{+240}$ \\
${ }^{208} \mathrm{~Pb}\left({ }^{54} \mathrm{Cr}, 1 n\right){ }^{261} \mathrm{Sg}$ & 106 & 262 & 202.01 & $2520_{-253}^{+253}$ \\
${ }^{209} \mathrm{Bi}\left({ }^{54} \mathrm{Cr}, 1 n\right){ }^{262} \mathrm{Bh}$ & 107 & 263 & 205.81 & $163_{-34}^{+34}$ \\
$\left.{ }^{208} \mathrm{~Pb}\left({ }^{58} \mathrm{Fe}, 1 n\right)\right)^{265} \mathrm{Hs}$ & 108 & 266 & 219.27 & $69_{-12}^{+12}$ \\
\hline \hline
\end{tabular}

though there are more measured data in the literature. Accordingly, the selected reaction systems and the corresponding maximum production cross-sections are summarized in Tab. I]

As a whole, it is clearly demonstrated that the maximal production cross-section drops considerably with increasing atomic number from $Z=104$ to $Z=108$, whereas the experimental uncertainty gradually rises due to increasing difficulty in performing such measurements.

\section{Uncertainty propagation}

To propagate input uncertainties through the model so as to quantify the output uncertainty, we use a MonteCarlo simulation as recommended in Ref. [51, for calculating the mean value $\mu_{Y}$ and the standard deviation $u(Y)$ associated with the model response. More concretely, supposing that one has a set of samples for the input random vector, namely $\left\{\boldsymbol{x}_{1}, \boldsymbol{x}_{2}, \ldots, \boldsymbol{x}_{S}\right\}$, the random model function is then evaluated for each of them as follows:

$$
y_{r}=\mathcal{M}\left(\boldsymbol{x}_{r}\right), r=1, \ldots, S .
$$

Thus, the usual statistical estimators of these quantities are simply given by

$$
\begin{aligned}
\mu_{Y} & =\frac{1}{S} \sum_{r}^{S} \mathcal{M}\left(\boldsymbol{x}_{r}\right) \\
u^{2}(Y) & =\frac{1}{S-1} \sum_{r}^{S}\left[\mathcal{M}\left(\boldsymbol{x}_{r}\right)-\mu_{Y}\right]^{2} .
\end{aligned}
$$

As regards the sample size, it can be approximately determined by checking the stability and convergence of the calculated result. At a $95 \%$ confidence level, the value of $S$ is determined to be 40000 , which would give a relative numerical uncertainty of less than $1 \%$. In the following study, the confidence level is kept fixed at $95 \%$ as usual.

To obtain the corresponding confidence interval, first, one has to estimate the cumulative distribution function (CDF) related to the output quantity, namely

$$
F_{Y}(y)=\int_{-\infty}^{y} f_{Y}(t) \mathrm{d} t .
$$

The estimated CDF, denoted by $\hat{F}_{Y}(y)$, can be obtained as follows 51:

- Sorting the values $\left\{y_{r}\right\}$ of the output quantity provided by the Monte-Carlo simulation into increasing order. The sorted values are denoted by $\left\{y_{(r)}\right\}$.

- Assigning uniform cumulative probabilities $p_{r}=$ $(r-1 / 2) / S$ to the ordered values.

Finally, once the estimated CDF has been constructed, it is possible to determine the endpoints that define the required confidence interval. In the case of symmetric output distributions, the $95 \%$ confidence interval is determined by the 0.025 - and 0.975 -quantiles. If the output distribution is asymmetric the shortest confidence interval should be adopted. It generally does not match with the 0.025- and 0.975-quantiles anymore and can be obtained numerically from the estimated CDF. For details on this approximation and the practical algorithm, the reader is referred to Ref. [51].

\section{Summary}

In the following, we calculate the empirical formation probability, $\bar{P}_{\text {form }}\left(\delta E^{*}, \beta, E_{d}, \sigma_{\text {exp }}^{1 n}\right)$ defined in Eq. 18 , and perform an uncertainty analysis that is essentially based upon the recommendations given in Ref. [51] which serves as the international standard for expression of uncertainty.

Regarding the PDFs for input parameters, they are summarized as follows:

- $\sigma_{\exp }^{1 n}$ is assumed to be normally distributed.

- $\delta E^{*} \sim U(2,4)$ in $\mathrm{MeV}$. Note that over each value of $\delta E^{*}$, the survival probability is averaged (cf. Eq. (16).

$$
\begin{aligned}
& \text { - } \beta \sim U(1.0,9.0) \text { in } \mathrm{zs}^{-1} . \\
& \text { - } E_{d} \sim U(13.0,25.0) \text { in } \mathrm{MeV} .
\end{aligned}
$$

As previously discussed, the above ranges of values of the last two parameters are considered under the pessimistic hypothesis, which simply means that they seem somewhat large but can cover most of the parameter values that can be found in the literature. Moreover, it would be instructive to take a look into the effects of using some narrower ranges of values on the final results. This is the optimistic case, where the values of $\beta$ and $E_{d}$ are supposed to lie within the following ranges:

$$
\begin{aligned}
& \text { - } \beta \sim U(3.0,7.0) \text { in } \mathrm{zs}^{-1} . \\
& \text { - } E_{d} \sim U(16.0,22.0) \text { in } \mathrm{MeV} \text {. }
\end{aligned}
$$

In addition to the input parameters, the effects of changing model functions on the calculated results should also be examined. Here, we mainly focus on some theoretical corrections, namely the collective enhancement 


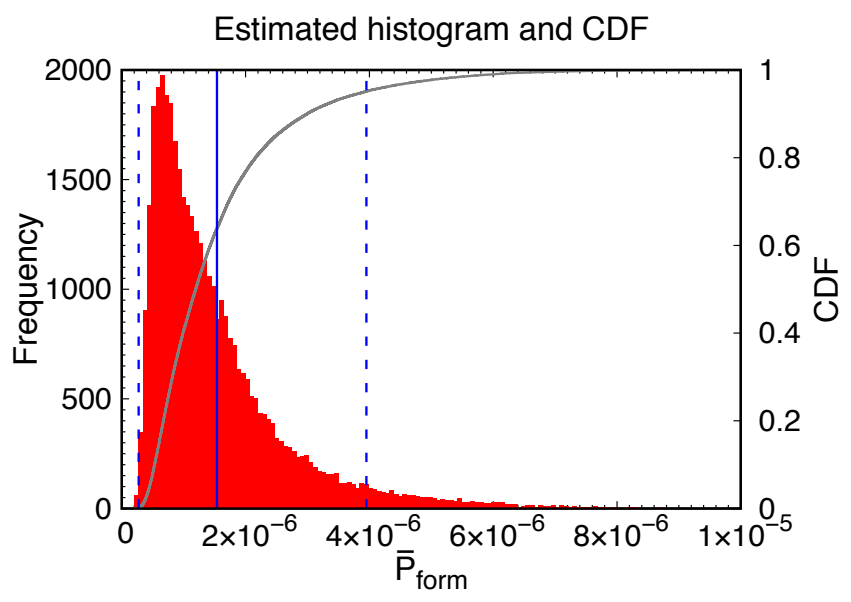

FIG. 5. [Color online] Estimated distribution of the empirical formation probability for the reaction ${ }^{208} \mathrm{~Pb}\left({ }^{58} \mathrm{Fe}, 1 n\right){ }^{265} \mathrm{Hs}$. The solid line represents the mean value and the dotted lines refer to the lower and upper bounds of the $95 \%$ confidence interval, respectively. The cumulative distribution function (CDF) of the empirical formation probability used to define the confidence interval is indicated.

and the Kramers-Strutinsky factors, as well as the fissionbarrier models, the level-density parameters and two simple methods for estimating capture cross-sections, which are considered to be the main physical ingredients included in the model.

It should be recalled that the default parameter values and models were concretely presented in a recent paper on the description of KEWPIE2 [3]. To briefly summarize, in the present study, the empirical barrierdistribution method [10, 55] for estimating the capture cross-section, the Tōke-Świątecki model [36] for the leveldensity parameter, the Thomas-Fermi model [56] for calculating fission barriers, and the collective enhancement and Kramers-Strutinsky factors [33, 34, 57, 58] have been considered by default.

The Monte-Carlo approach is employed to propagate input distributions through the model, as the uncertainty associated with each of the input parameters is considerably large. In the present work, the GSL scientific library [59] was employed to generate uniform and normal random numbers for input distributions.

\section{RESULTS AND DISCUSSION}

In this section, we start by showing an illustrative example for the cold-fusion reaction leading to the formation of element $\mathrm{Hs}$, namely ${ }^{208} \mathrm{~Pb}\left({ }^{58} \mathrm{Fe}, 1 n\right){ }^{265} \mathrm{Hs}$, at its optimum energy. Then, a systematic study is carried out and discussed.
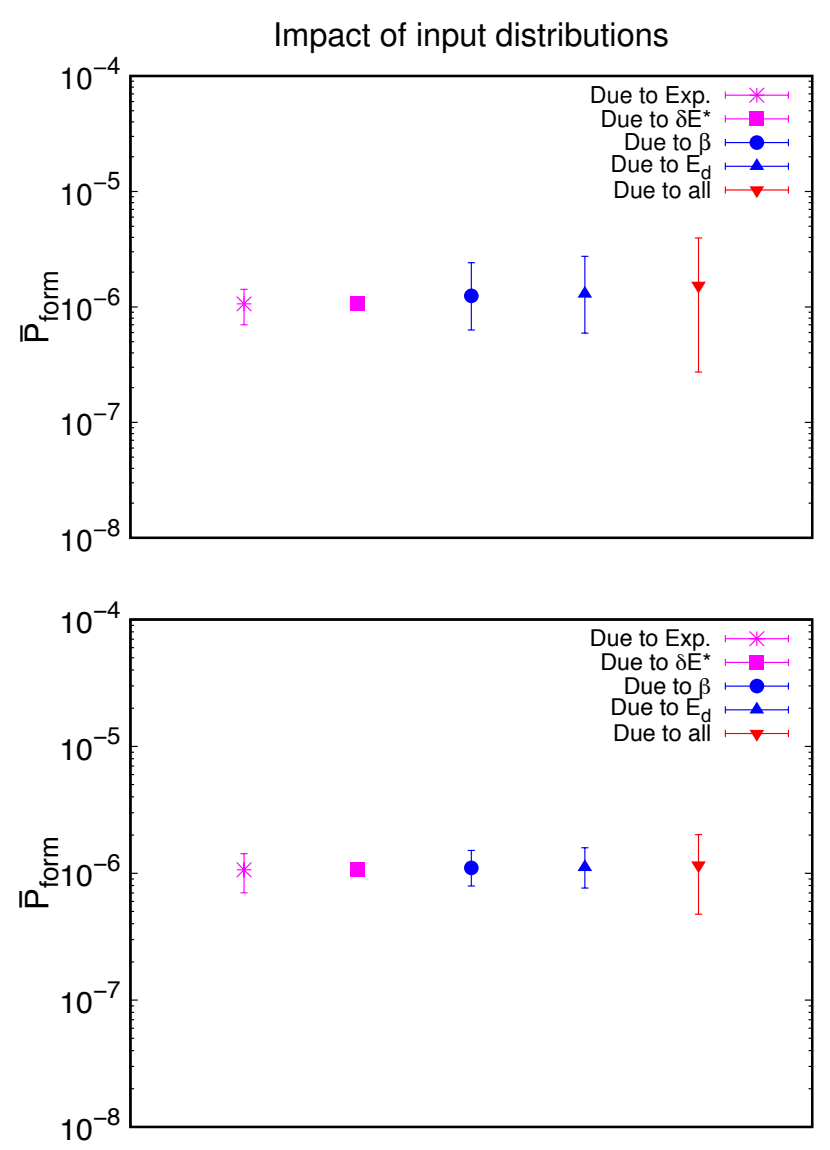

FIG. 6. [Color online] Impact of input distributions on uncertainty associated with the empirical formation probability. The pessimistic case is considered in the upper panel and the optimistic input distributions for $\beta$ and $E_{d}$ in the lower one. See text for details.

\section{A. Influence of input distributions}

Fig. 5 illustrates the estimated distribution related to the empirical formation probability obtained by means of the KEWPIE2 code together with available production cross-sections (cf. Eq. [18)). It should be mentioned that all the input parameters were considered under the pessimistic hypothesis. In this case, the shape of the distribution appears to be highly skewed to the left, that is, it has a long tail on the right hand side of the distribution and thus results in a quite asymmetric confidence interval. The mean value is hence located on the right-hand side of the peak value. Fig. 5 also displays the corresponding CDF used to determine both lower and upper bounds of the $95 \%$ confidence interval.

Separate uncertainty contributions coming from each of the input distributions are displayed in Fig. 6. Each point stands for the mean value of the empirical formation probability, together with its $95 \%$ confidence interval. First, the contribution of the experimental uncertainty is represented by the first point on the left. The 
upper bound of the confidence interval is higher than the lower one by a factor of about 2 . The following three points are connected with the energy loss in the target, the reduced friction parameter $\beta$ and the shell-damping energy $E_{d}$, respectively. Overall, it can be clearly noticed that the theoretical contributions are dominant compared to the experimental ones. This is simply due to the fact that $\beta$ and $E_{d}$ are closely related to the fission process that is known to be the dominating decay channel for SHE. It should also be mentioned that the relative uncertainty of $\beta$ is about $46 \%$, which appears to be much larger than that of $E_{d}$ estimated to be about $18 \%$. Put differently, this means that uncertainty related to the empirical formation probability would be more sensitive to the shell-damping energy. Finally, when including all input distributions, the total confidence interval spans slightly more than one order of magnitude.

When reducing the intervals of the two dominant parameter values by $50 \%$, that is, under the optimistic hypothesis, Fig. 6 clearly demonstrates that the total uncertainty is also decreased by almost the same factor. This result also indicates that further constraints on these two critical model parameters would be able to reduce the total uncertainty connected with the empirical formation probability.

\section{B. Influence of models}

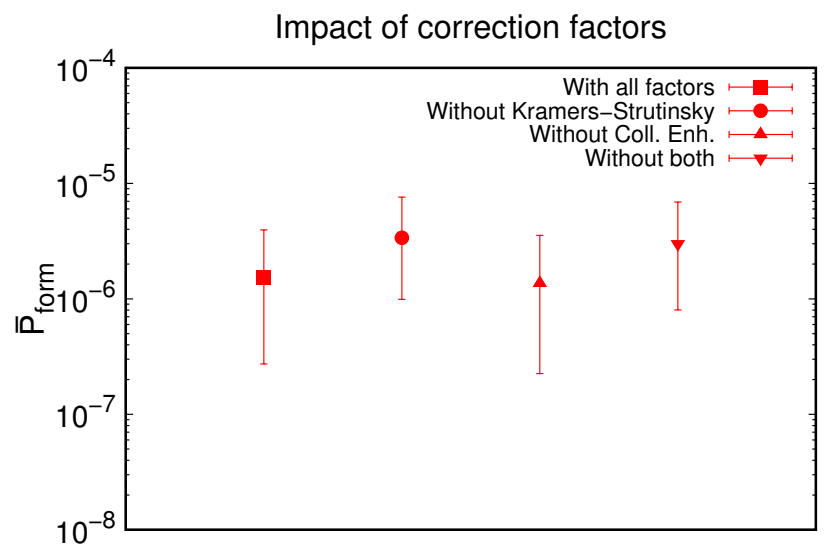

FIG. 7. [Color online] Impact of correction factors on the empirical formation probability. Note that the uncertainty interval is due to all parameters under the pessimistic hypothesis.

We then look into the impact of changing models on the empirical formation probability.

Fig. 7 illustrates how correction factors, namely the Kramers-Strutinsky and collective enhancement ones, affect the mean value of the empirical formation probability. Removing the Kramers-Strutinsky factor, it is readily seen that the mean value slightly increases by a factor

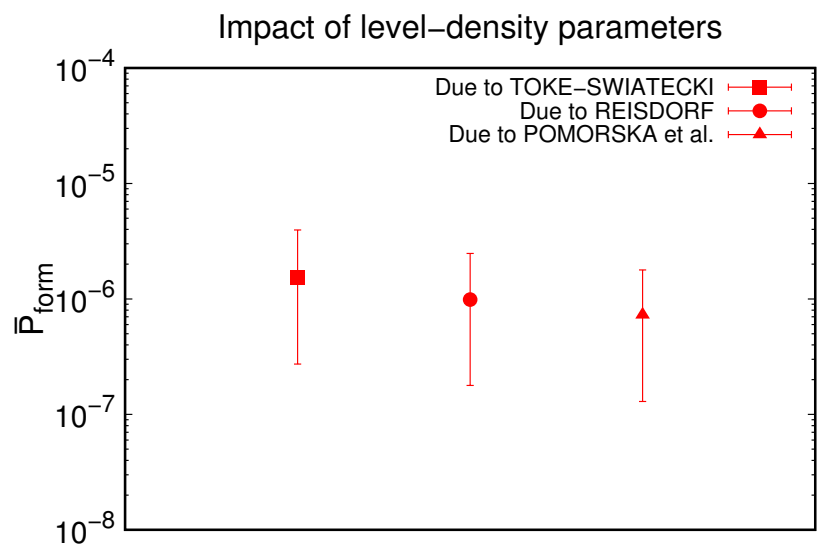

FIG. 8. [Color online] Impact of level-density parameters on the empirical formation probability. Uncertainty interval is due to all parameters under the pessimistic hypothesis.

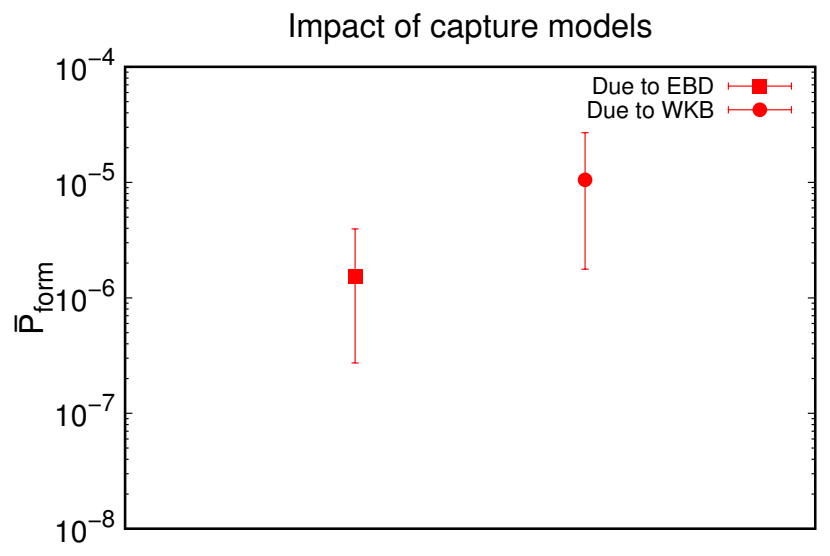

FIG. 9. [Color online] Impact of capture models on the empirical formation probability. Uncertainty interval is due to all parameters under the pessimistic hypothesis.

of about 2, whereas it becomes a bit lower after removing the collective enhancement factor. It should also be noted that the uncertainty amplitudes do not remain the same due to the fact that $\beta$ is jointly removed along with the Kramers-Strutinsky factor so that the output distribution becomes narrower.

Fig. 8 shows the effects of changing level-density parameter formulas on the mean value of the empirical formation probability and the associated confidence interval. As already mentioned, in addition to the default model of Tōke and Świątecki [36], we also selected the Reisdorf 24 and Pomorska et al. models [37. Here, one can notice that the mean value of the empirical probability decreases at most by a factor of around 2, which appears to be negligible compared to the total uncertainty interval. As a whole, the lengths of their confidence intervals seem to remain the same.

Fig. 9 tells us how capture models affect the mean value 


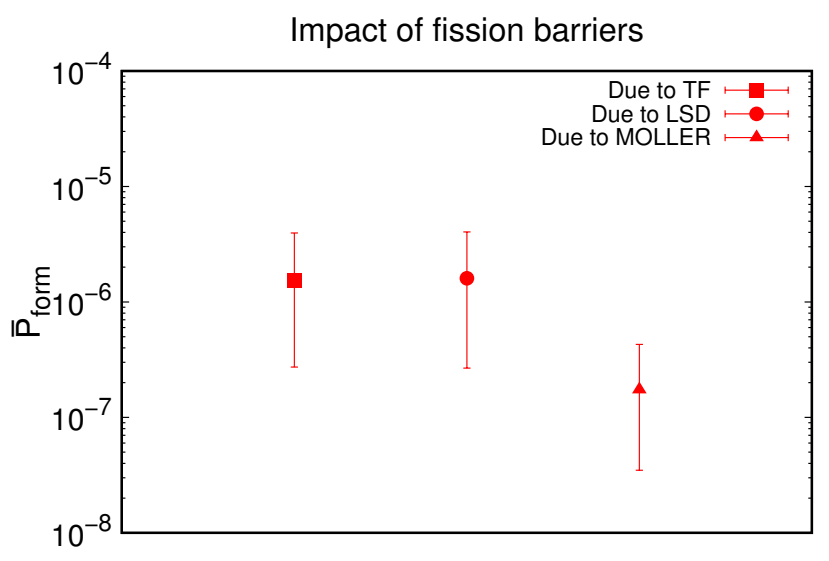

FIG. 10. [Color online] Impact of fission barriers on the empirical formation probability. Uncertainty interval is due to all parameters under the pessimistic hypothesis.

and confidence interval of the empirical formation probability. Here, the empirical barrier-distribution (EBD) method [10, 23] and the WKB approximation [24, 25], were considered. As can be seen in Fig. 9, the results based upon these two simple capture models seem to differ by a factor of about 7 , that is, less than one order of magnitude. However, special attention should always be drawn to the capture step because calculated crosssections could differ by one order of magnitude, especially in the case of deformed colliding nuclei due to nuclear structure effects.

Fig. 10 demonstrates how the mean value and the confidence interval associated with the empirical formation probability evolve with various fission-barrier models. Here, three different models were considered. Two of them are based on a commonly used approximation with a macroscopic part calculated with the ThomasFermi (TF) model [56] or the Lublin-Strasbourg Drop (LSD) [60, to which a shell-correction table 61] is added. The third model is Möller et al's microscopic-macroscopic model [32]. It is thus observed that the mean values can differ by almost one order of magnitude that is comparable to the total uncertainty interval. This is mainly due to the fact that nuclear fission is known to be the dominant decay channel for heavy nuclei. A small change in fission barrier can give rise to a significant variation in the calculated survival probability. It is worth noticing that if we had taken into account all fission barriers available in the scientific literature, the impact would have been larger.

\section{An extended comparison}

So far, we have illustrated an uncertainty analysis for a special cold-fusion reaction. In this subsection, an extended study is presented for the whole set of cold-fusion reactions listed in Tab. I.

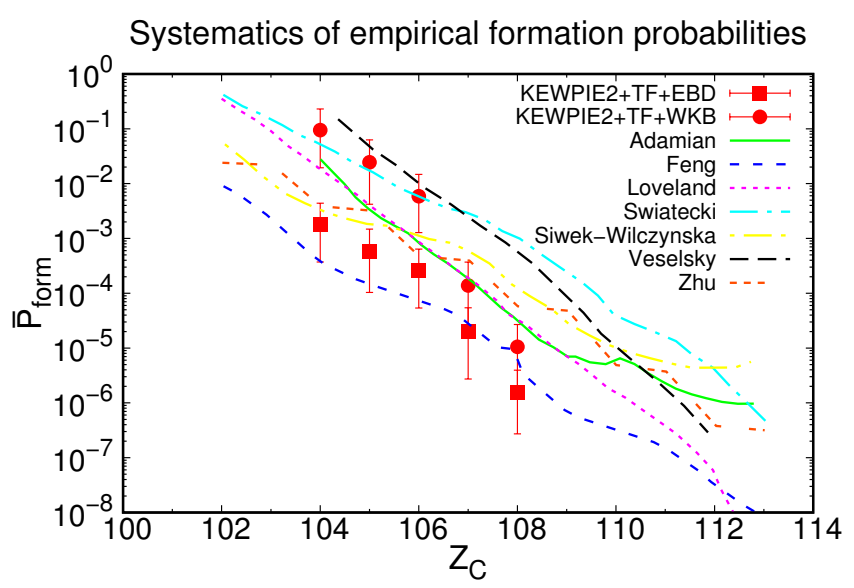

FIG. 11. [Color online] Systematic comparison of the calculated mean values of the empirical formation probability for the cold-fusion reactions leading to the synthesis of the elements from $Z=104$ to $Z=108$ (cf. Tab. I). The deduced formation probabilities are based upon two simple capture models (see text). Lines correspond to theoretical predictions from Refs. [10, 16, 20, 62] as in Fig. 2,

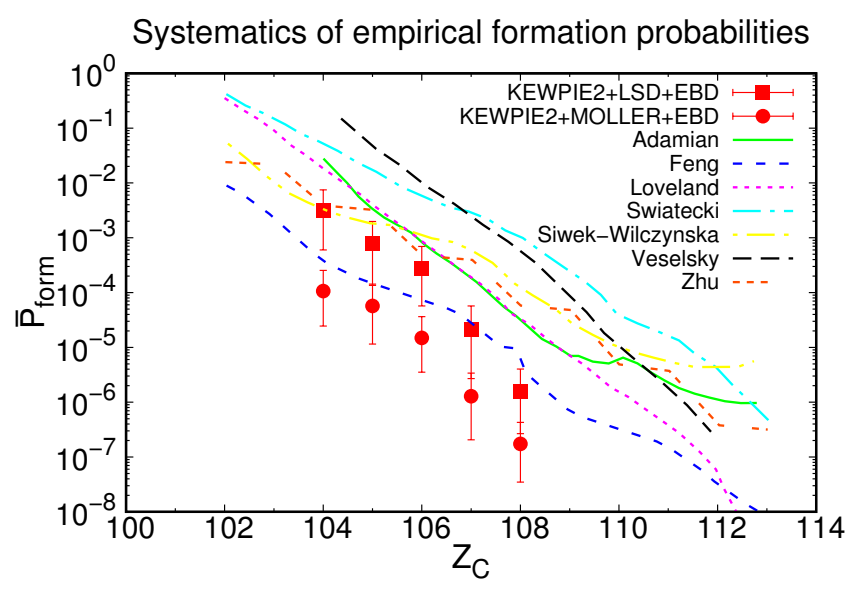

FIG. 12. [Color online] Same as Fig. 11 but the deduced formation probabilities are based upon two different fissionbarrier models (see text).

Two different cases related to the empirical formation probabilities and the associated $95 \%$ confidence intervals for the selected cold-fusion reactions leading to the synthesis of SHE ranging from $Z=104$ to $Z=108$ are shown in Figs. 11 and 12 . The former displays calculated results with two simple capture models and the latter shows those based upon two extreme fission-barrier models. It should be kept in mind that the uncertainty interval is always related to all input distributions within the pessimistic hypothesis.

First, one can immediately notice that the discrepancy due to the capture step can even reach two orders of magnitude for the lightest reaction system, but it gradually declines as the atomic number of the compound system 


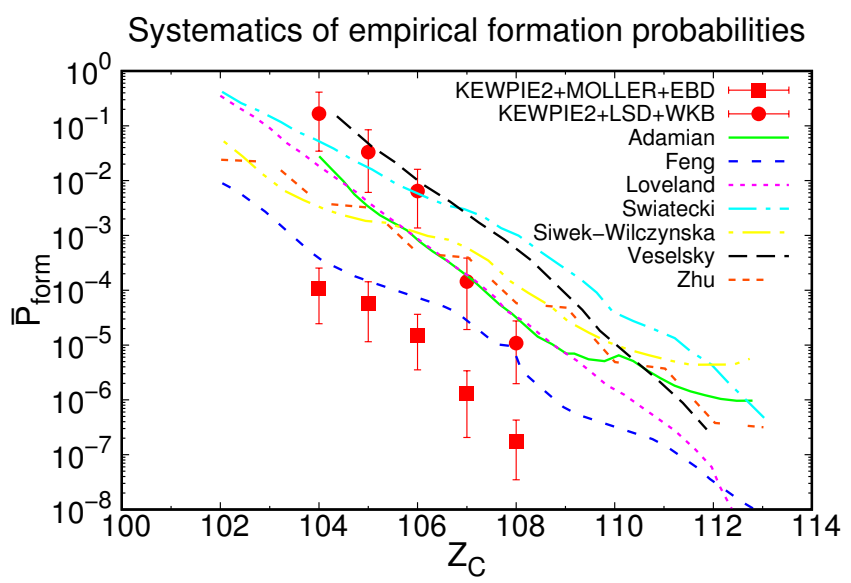

FIG. 13. [Color online] Same as Fig. 11 but the extreme case is presented for both capture and fission-barrier models so as to have the largest discrepancy between the empirical formation probabilities.

goes up. This is due to the fact that, as the system becomes heavier, the incident energy for the one-neutron channel is approaching to the Coulomb barrier so that uncertainty associated with the capture model because of sub-barrier coupled-channels effects should be gradually reduced. To this extent, the uncertainty remaining in the estimated capture cross-section could also have a significant impact on the final results, but fortunately it can be directly measured and thus be constrained. Here, it should be kept in mind that we only considered two simple capture models in our calculations, whereas many other more sophisticated and accurate ones are currently available. Accordingly, one would expect that uncertainty associated with the capture model can be reduced either theoretically or experimentally.

Regarding the impact of fission barriers, as displayed in Fig. 12, the discrepancy practically seems to remain the same. Overall, the mean values based upon two extreme fission-barrier models with the same shell correction energy can differ by about one order of magnitude, which is comparable to the lengths of their corresponding uncertainty intervals. Unlike in the case of capture models, since the fission-barrier height cannot be directly measured, it is unlikely that uncertainty would be reasonably reduced. To this extent, other methods should to be invented so as to constrain fission-barrier models. For instance, we recently started to consider Bayesian inference together with its possible application [63, 64.

Finally, it is also interesting to take a look into the extreme cases, that is, with two capture models and two extreme fission-barrier models. This is displayed in Fig. 13. As for some lighter systems, the maximal discrepancy can reach more than three orders of magnitude, whereas it gradually diminishes to around two orders of magnitude as the atomic number goes up. Considering the total uncertainty interval, the discrepancy between the calculated formation probabilities can be completely accommodated and hence, it appears unlikely to discriminate the various formation models. Put differently, as mentioned at the beginning of this paper, all fusion-evaporation models would be capable of reproducing the measured data, even though there are still serious ambiguities in the reaction mechanism for heavy-ion fusions.

\section{CONCLUSION AND PERSPECTIVES}

Although various models converge to experimental data when estimating excitation function of ER crosssections of super-heavy nuclei, a closer look at intermediate steps shows large discrepancies between them. It is especially the case for the formation step that is the lesser known part of the reaction. Consequently, when experimental data are not available, predictions diverge. The results obtained from the uncertainty analysis presented in this paper are helpful for comprehending the contradiction between theoretical formation probabilities and ER cross-sections. It was demonstrated that, although the formation probability is not yet quantitatively known, by somehow tuning the better-known factors, namely the capture cross-section and survival probability, one can still be able to obtain a reasonable fit to experimental data.

In this sense, the predictive power of fusionevaporation models remains quite limited in the case of super-heavy element synthesis. To assess them, it would be necessary to refine both the capture and fission-barrier models. In the former case, as previously mentioned, this can be done with the help of more accurate data as the capture cross-section can be directly measured, or using some more sophisticated capture models. In the latter case, however, there are still severe discrepancies between various types of calculations 31 and moreover, the fission-barrier height cannot be directly measured. Its extraction from experimental data is model dependent 63, 64.

How to constrain both formation models and fission barriers simultaneously? There is a crucial need for experiments dedicated to a better understanding of the fusion hindrance. The synthesis of the same nucleus by hindered and non-hindered reactions would be helpful as the decay part would be the same. From a theoretical point of view, there is a real need to decrease the number of free parameters. Thus, fission barriers and inner fusion barriers responsible for the fusion hindrance should be calculated within the same model. This generally requires microscopic or microscopic-macroscopic approaches [31.

\section{ACKNOWLEDGMENT}

The authors acknowledge JSPS and the FrenchJapanese International Associated Laboratory for $\mathrm{Nu}$ clear Structure Problems (LIA-FJNSP) for partial support of this work. Part of this work is supported by NSF 
of China under grant number 11275068 and 11547312 . The authors greatly appreciate the warm hospitality and support provided by GANIL and RCNP at Osaka Univ., which enabled them to work together. H. L. would like to thank the staff at Huzhou Univ. for their hospital- ity. This work is part of H. L.'s Ph.D. thesis which was supported by the Centre National de la Recherche Scientifique (CNRS) and the Conseil Régional de BasseNormandie.
[1] N. Bohr, Nature 137, 344 (1936)

[2] A. Marchix, PhD thesis, Université de Caen (2007), http://tel.archives-ouvertes.fr/tel-00197012/

[3] H. Lü, A. Marchix, Y. Abe and D. Boilley, Comp. Phys. Comm. 200, 381 (2016)

[4] C. Shen, G. Kosenko and Y. Abe, Phys. Rev. C 66, 061602(R) (2002)

[5] R. Bass, Phys. Rev. Lett. 39, 265 (1977)

[6] Y. Abe, D. Boilley, B.G. Giraud and T. Wada, Phys. Rev. E 61, 1125 (2000)

[7] Y. Abe, D. Boilley, G. Kosenko, J.D. Bao, C. Shen, B. Giraud and T. Wada, Prog. Theor. Phys. Supp. 146, 104 (2002)

[8] Y. Abe, B. Bouriquet, C. Shen and G. Kosenko, Nucl. Phys. A 722, C241 (2003)

[9] D. Boilley, Y. Abe and J.D. Bao, Eur. Phys. J. A 18, 627 (2003)

[10] W. J. Świa̧tecki, K. Siwek-Wilczyńska and J. Wilczyński, Phys. Rev. C 71, 014602 (2005)

[11] C. Shen, Y. Abe, D. Boilley, G. Kosenko and E. Zhao, Int. J. Mod. Phys. E 17, 66 (2008)

[12] D. Boilley, H. Lü, C. Shen, Y. Abe and B. G. Giraud, Phys. Rev. C 84, 054608 (2011)

[13] S. Hofmann, J. Phys. G: Nucl. Part. Phys. 42, 114001 (2015)

[14] R. S. Naik, W. Loveland, P. H. Sprunger, A. M. Vinodkumar, D. Peterson, C. L. Jiang, S. Zhu, X. Tang, E. F. Moore and P. Chowdhury, Phys. Rev. C 76, 054604 (2007)

[15] B. Bouriquet and Y. Abe and G. Kosenko, Eur. Phys. J. A 22, 9 (2004)

[16] M. Veselskỳ, Acta Phys. Slov. 49, 101 (1999)

[17] G.G. Adamian, N.V. Antonenko and W. Scheid, Nucl. Phys. A 678, 24 (2000)

[18] Z.-Q. Feng, G.-M. Jin, J.-Q. Li and W. Scheid, Phys. Rev. C 76, 044606 (2007)

[19] W. Loveland, Phys. Rev. C 76, 014612 (2007)

[20] K. Siwek-Wilczyńska, I. Skwira-Chalot and J. Wilczyński, Int. J. Mod. Phys. E 16, 483 (2007)

[21] Editors, Phys. Rev. A83, 040001 (2011)

[22] W. Loveland, Eur. Phys. J. A 51, 120 (2015)

[23] K. Siwek-Wilczyńska and J. Wilczyński, Phys. Rev C 69, 024611 (2004)

[24] W. Reisdorf, Z. Phys. A300, 227 (1981)

[25] W. Reisdorf, F. Heßsberger, K. Hildenbrand, S. Hofmann, G. Münzenberg, K.-H. Schmidt, J. Schneider, W. Schneider, K. Sümmerer, G. Wirth, J.V. Kratz and K. Schlitt, Nucl. Phys. A 438, 212 (1985)

[26] V.F. Weisskopf, Phys. Rev. 52, 295 (1937)

[27] V.F. Weisskopf and D.H. Ewing, Phys. Rev. 57, 472 (1940)

[28] H. Delagrange, C. Grégoire, F. Scheuter and Y. Abe, Z. Phys. A 323, 437 (1986)
[29] I. Dostrovsky, Z. Fraenkel and G. Friedlander, Phys. Rev. 116,683 (1959)

[30] N. Bohr and J.A. Wheeler, Phys. Rev. 56, 426 (1939)

[31] A. Baran, M. Kował, P.-G. Reinhard, L.M. Robledo, A. Staszczak and M. Warda, Nucl. Phys. A 944, 442 (2015)

[32] P. Möller, A.J. Sierk, T. Ichikawa, A. Iwamoto, R. Bengtsson, H. Uhrenholt and S. Åberg, Phys. Rev. C 79, 064304 (2009)

[33] H. A. Kramers, Physica 7, 284 (1940)

[34] V. M. Strutinsky, Phys. Lett. B 47, 121 (1973)

[35] M. Grossjean and H. Feldmeier, Nucl. Phys. A 444, 113 (1985)

[36] J. Töke and W. J. Świątecki, Nucl. Phys. A 372, 141 (1981)

[37] B. Nerlo-Pomorska, K. Pomorski and J. Bartel, Phys. Rev. C 74, 034327 (2006)

[38] A.V. Ignatyuk, G.N. Smirenkin and A.S. Tishin, Yad. Fiz. 21, 485 (1975) (Sov. J. Nucl. Phys. 21, 255 (1975))

[39] M.C. Kennedy and A. O'Hagan, J. R. Stat. Soc. Series B Stat. Methodol. 63, 425 (2001)

[40] D. Hilscher and H. Rossner, Ann. Phys. Fr. 17, 471 (1992)

[41] Y. Abe, S. Ayik, P.-G. Reinhard and E. Suraud, Phys. Rep. 275, 49 (1996)

[42] P.C. Rout, D.R. Chakrabarty, V.M. Datar, S. Kumar, E.T. Mirgule, A. Mitra, V. Nanal, S.P. Behera and V. Singh, Phys. Rev. Lett. 110, 062501 (2013)

[43] B. Jurado, C. Schmitt, K.-H. Schmidt, J. Benlliure and A.R. Junghans, Nucl. Phys. A 747, 14 (2005)

[44] J.P. Lestone and S.G. McCalla, Phys. Rev. C 79, 044611 (2009)

[45] S.G. McCalla and J.P. Lestone, Phys. Rev. Lett. 101, 032702 (2008)

[46] Y. Aritomo and S. Chiba, Phys. Rev. C 88, 044614 (2013)

[47] R. Capote, M. Herman, P. Obložinský, P.G. Young, S. Goriely, T. Belgya, A.V. Ignatyuk, A.J. Koning, S. Hilaire, V.A. Plujko, M. Avrigeanu, O. Bersillon, M.B. Chadwick, T. Fukahori, Z. Ge, Y. Han, S. Kailas, J. Kopecky, V.M. Maslov, G. Reffo, M. Sin, E.Sh. Soukhovitskii and P. Talou, Nucl. Data Sheets 110, 3107 (2009)

[48] C. Stodel, private communication

[49] C.E. Shannon, Bell System Technical Journal 27, 379 (1948)

[50] E.T. Jaynes, Phys. Rev. 106 (1957) 620; Phys. Rev. 108, $171(1957)$

[51] BIPM, IEC, IFCC, ILAC, ISO and IUPAC, Joint Committee for Guides in Metrology 101 (2008)

[52] G. Münzenberg, S. Hofmann, F.P. Heßberger, W. Reisdorf, K.-H. Schmidt, J.H.R. Schneider, P. Armbruster, C.C. Sahm and B. Thuma, Z. Phys. A 300, 107 (1981)

[53] S. Hofmann and G. Münzenberg, Rev. Mod. Phys. 72, $733(2000)$

[54] S. Hofmann, F.P. Heßberger, D. Ackermann, S. Antalic, P. Cagarda, B. Kindler, P. Kuusiniemi, M. Leino, B. 
Lommel, O.N. Malyshev, R. Mann, G. Münzenberg, A.G. Popeko, S. Śaro, B. Streicher and A.V. Yeremin, Nucl. Phys. A 734, 93 (2004)

[55] T. Cap, K. Siwek-Wilczyńska and J. Wilczyński, Phys. Rev. C 83, 054602 (2011)

[56] W. D. Myers and W. J. Świątecki, Phys. Rev. C 60, 014606 (1999)

[57] A.R. Junghans, M. de Jong, H.-G. Clerc, A.V. Ignatyuk, G.A. Kudyaev and K.-H. Schmidt, Nucl. Phys. A 629, 635 (1998)

[58] V.I. Zagrebaev, Y. Aritomo, M.G. Itkis, Yu. Ts. Oganessian and M. Ohta, Phys. Rev. C 65, 014607 (2001)
[59] B. Gough, GNU scientific library reference manual, Network Theory Ltd. (2009)

[60] F. A. Ivanyuk and K. Pomorski, Phys. Rev. C 79, 054327 (2009)

[61] P. Möller, J.R. Nix, W.D. Myers and W. J. Świątecki, At. Data. Nucl. Data Tables 59, 185 (1995)

[62] L. Zhu, W.-J. Xie and F.-S. Zhang, Phys. Rev. C 89, $024615(2014)$

[63] H. Lü and D. Boilley, EPJ Web of Conferences 62, 03002 (2013)

[64] H. Lü, PhD thesis, Université de Caen Normandie (2015), http://hal.in2p3.fr/tel-01235448 